\title{
Modelling of thermally enhanced erosion of beryllium
}

\author{
K. Schmid * \\ Max-Planck-Institut für Plasmaphysik, Boltzmannstraße 2, D-85748 Garching b. München Germany \\ M. Baldwin, R. Doerner \\ UCSD, Center For Energy Research, 460 EBU II 9500, Gilman Drive, La Jolla CA 92093 USA
}

\begin{abstract}
This paper presents two concurring models for the thermally enhanced erosion of metals. The modelling particularly deals with the erosion of beryllium by a helium plasma as an example system. Molecular Dynamics (MD) simulations are used to reduce the number of free parameters in the models. A model of sublimation of ad-atoms created during ion impact was earlier proposed as an explanation of thermally enhanced erosion. Using MD calculations the parameter space for this model was reduced to a single free parameter, the areal surface defect density $\delta_{D e f}$. Using the reduced parameter space a very low $\delta_{D e f}$ has to be assumed in order to reproduce the experimental observations. Therefore a new model is proposed here that is very similar to the ad-atom model but is based on a different mechanism to create weakly bonded surface atoms. The paper shows that inclusion of He atoms during exposure to high flux $\left(10^{22} \mathrm{~m}^{-2} \mathrm{~s}^{-1}\right)$ of low energy $\mathrm{He}(50 \mathrm{eV})$ leads to the formation of weakly bonded atoms in the surface. The comparison of both models with experimental data and their applicability to other projectile/target systems is discussed.
\end{abstract}

* Corresponding Author

Tel: +49 8932992228

Fax: +49 8932992279

E-mail: Klaus.Schmid@ipp.mpg.de 


\section{Introduction}

The sputtering of materials by energetic projectiles is a process that has been studied extensively both experimentally [1] and through computer simulations [2] since the fundamental works of Nils Bohr [3] in 1948. Sputtering by particle impact plays an important role in a variety of scientific fields ranging from semiconductor surface processing to plasma wall interaction in magnetic fusion confinement devices.

The currently accepted picture of surface erosion through energetic particle bombardment is that the total erosion rate is given by the sum of the physical sputtering rate and the sublimation rate of the material. The theory of physical sputtering is well understood [4] and most importantly the sputtering yield does not depend on the surface temperature. The sublimation rates are readily available for most elements and depend only on the temperature and not on the particle flux. Thus applying this current picture one would expect the total erosion rate to be constant at low temperatures, purely determined by the physical sputtering rate. At high temperatures where the sublimation rate becomes comparable to the physical sputtering rate, one would expect an exponential increase with temperature, purely governed by the material sublimation rate.

However, already nearly 20 years ago it was found $[5,6]$ that graphite, if exposed to $\mathrm{keV}$ noble gas or D ions, exhibits an exponential increase with temperature at $1100 \mathrm{~K}$ which could not be explained by normal sublimation of graphite which is still negligible at $1100 \mathrm{~K}$. This effect was called radiation enhanced sublimation (RES). The explanation [7] of RES is based on the formation and sublimation of $\mathrm{C}$ interstitials through energetic particle impact. These interstitials once generated are very mobile and can diffuse to the surface at elevated temperatures where they sublime easily due to their low surface binding energy.

As pointed out in [8] a similar effect was found in recent experiments during exposure of metals (some in liquid state) to a high flux low energy particle flux from a plasma. The model explanation given in [8] was that surface atoms were raised on top of the surface due to the energetic particle impact resulting weakly 
bonded ad-atoms that would sublime at lower temperatures as the normal surface atoms. This model not only explained the experimental observations but also why so far thermally enhanced erosion of metals was not found during ion beam experiments. The reason for that is that in an ion beam experiment the particle fluxes are four orders of magnitude lower than in a plasma experiment. Thus the ad-atom creation rate is also lower by four orders of magnitude. Thus it does not contribute significantly to the erosion flux. Therefore erosion in ion beam experiments is dominated by conventional sublimation at elevated temperatures. While the ad-atom model was very successful in explaining the experimental data a question remained: Is the lifetime of these ad-atoms long enough to sublime or do they recombine with surface defects long before they sublimate? To answer this question an analytical expression for the erosion yield was derived. Then estimates for the parameters in this analytical expression were obtained from molecular dynamics calculations. This procedure left the areal surface defect density $\delta_{D e f}$ as the only free parameter. The model was then fitted to experimental data for the thermally enhanced erosion of Be by a He plasma using $\delta_{D e f}$ as a free parameter.

Since the required $\delta_{D e f}$ turned out to be very low for a realistic target surface a different model is also discussed in this paper. It is very similar to the ad-atom model but is based on a different mechanism to weaken the surface binding energy. This "Inclusion" model is based on the formation of weakly bonded surface atoms due to inclusion of the implanted species below the surface. After developing an analytical expression for the erosion yield and obtaining estimates for all free parameters from MD calculations the "Inclusion" model reproduced the experimental data without free parameters.

The paper shows the derivation of the analytical expressions for the erosion yields for both models and how the free parameters were estimated from MD calculations. Then, using the calculated parameters, it will be discussed how the models can be fitted to experimental data and whether or not the required values for the remaining free parameters are of reasonable order of magnitude. 


\section{Experimental observations}

In Fig. 1 the erosion yield as a function of temperature is shown for a solid Be target exposed to D and He plasmas respectively as measured in the PISCES-B experiment. The sample was biased to $-50 \mathrm{~V}$ in both cases. The erosion yield was calculated by first subtracting the conventional sublimation flux from the spectroscopically measured Be flux off the sample and then dividing the result by the incident flux from Langmuir probe measurements. The details of the experiment and the PISCES-B setup can be found in [8]. For both D and He plasmas the erosion yield increases exponentially for temperatures above 1100 $K$. The relative increase in the erosion flux from the physical sputtering base level measured at low temperatures $\ll 1100 \mathrm{~K}$ is approximately a factor 30 in both cases.

\section{Analytical models}

To compare the models to the experimental data in Fig. 1 analytical expression are required for the total Be erosion yield $Y_{B e}$ at/ion. The principal terms for $Y_{B e}$ are the same for both models and can be summarized in equation 1:

$$
\begin{aligned}
Y_{B e} & =Y_{B e}^{\text {Phys }}+\frac{1}{\Gamma}\left(\Phi_{S u b}(T)+\Phi_{\text {Sub }}^{\text {Def }}(T)\right) \\
Y_{B e}^{\text {Phys }} & =\text { physical erosion yield } \\
\Gamma= & \text { incident flux } m^{-2} s^{-1} \\
\Phi_{\text {Sub }}(T)= & \text { normal sublimation flux } m^{-2} s^{-1} \\
\Phi_{\text {Sub }}^{\text {Def }}(T)= & \text { sublimation flux due to weakly } \\
& \text { bonded surface defects } m^{-2} s^{-1}
\end{aligned}
$$

The Be sputter yield $Y_{B e}^{P h y s}=0.02$ was calculated using the Binary Collision Approximation (BCA) Monte Carlo code TRIDYN [9]. Since in PISCES-B the ion temperature $(\approx 3 \mathrm{eV})$ is much smaller than the bias voltage, a mono-energetic beam of $50 \mathrm{eV}{ }^{4} \mathrm{He}$ incident normal to the Be target surface was assumed in the 
TRIDYN calculation. The sublimation fluxes $\Phi(T)$ as a function of temperature $\mathrm{T}$ in equation 1 can be calculated from equation 2 :

$$
\begin{aligned}
\Phi_{\text {Sub }}= & \delta \xi(T) \\
\xi(T)= & \xi_{0} e^{-\frac{E_{B}}{k_{B} T}} s^{-1} \\
\delta= & \text { areal density } \\
& \text { of sublimating species } m^{-2} \\
\xi_{0}= & \text { exponential pre-factor } s^{-1} \\
E_{B}= & \text { surface binding energy } \\
& \text { of sublimating species } \mathrm{eV}
\end{aligned}
$$

The parameters $\xi_{0}$ and $E_{B}$ were calculated from a fit to sublimation fluxes from [10] using the areal density of $\mathrm{Be}\left(2.5 \cdot 10^{22} \mathrm{~m}^{-2}\right)$ for $\delta$.

The two models only differ in the $\Phi_{\text {Sub }}^{\text {Def }}$ term which depends on the areal density of the respective weakly bonded surface defect and its sublimation rate which in turn is mainly governed by the defect's reduced surface binding energy $E_{B}$. For the ad-atom model the areal density of ad-atoms in equilibrium can be calculated from the following rate equation

$$
\begin{aligned}
\frac{d \delta_{A d}}{d t} & =\Gamma \gamma-\xi_{R e c} \delta_{A d}-\xi_{S u b, A} \delta_{A d} \\
\delta_{A d} & =\text { areal density of ad-atoms } m^{-2} \\
\gamma & =\text { ad-atom yield } \\
\xi_{\text {Rec }} & =\text { ad-atom recombination rate } s^{-1} \\
\xi_{S u b, A} & =\text { ad-atom sublimation rate } s^{-1}
\end{aligned}
$$

In equilibrium $\frac{d \delta_{A d}}{d t} \equiv 0$ and thus $\delta_{A d}$ can be calculated from equation 5 :

$$
\delta_{A d}=\frac{\Gamma \gamma}{\xi_{R e c}+\xi_{S u b, A}}
$$


and the sublimation flux of ad-atoms $\Phi_{S u b, A}^{D e f}$ evaluates to

$$
\begin{aligned}
\Phi_{S u b, A}^{D e f} & =\delta_{A d} \xi_{S u b, A} \\
& =\xi_{S u b, A} \frac{\Gamma \gamma}{\xi_{R e c}+\xi_{S u b}, A}
\end{aligned}
$$

The ad-atom sublimation rate $\xi_{S u b, A}$ in equation 6 can be calculated from equation 3 using the smaller surface binding energy $E_{B, A}$ of the ad-atoms. To calculate the ad-atom recombination rate $\xi_{R e c}$ in equation 6 the model assumes that recombination happens primarily at surface steps where no activation energy for the recombination process is necessary. The ad-atom recombination rate depends on how quickly they diffuse to on the surface and the average distance between defects. Given the diffusion coefficient $\mathrm{D}(\mathrm{T})$ the distance $\Delta x$ an adatom travels on average during its lifetime $\tau=\frac{1}{\xi_{R e c}}$ can be calculated from the Einstein relation

$$
\begin{aligned}
\Delta x= & \sqrt{4 D \tau} \\
D(T)= & D_{0} e^{\frac{-E_{D}}{k_{B} T}} \\
D_{0}= & \text { exponential pre-factor } \mathrm{cm}^{2} \mathrm{~s}^{-1} \\
E_{D}= & \text { activation energy } \\
& \text { for surface diffusion } \mathrm{eV}
\end{aligned}
$$

If $\Delta x$ is equal to the mean distance between two recombination sites described by the areal defect density $\delta_{D e f}$ the ad-atom is assumed to recombine and not contribute to the temperature dependent erosion. This can be written as

$$
\sqrt{4 D \tau}=\sqrt{\frac{1}{\delta_{D e f}}}
$$

and thus $\xi_{R e c}$ becomes

$$
\xi_{\text {Rec }}=4 D \delta_{D e f}
$$


Inserting equations 10 and 3 into equation 6 one obtains for the sublimation flux of ad-atoms:

$$
\Phi_{S u b, A}^{D e f}\left(\Gamma, T, \delta_{D e f}\right)=\frac{\Gamma \gamma}{\frac{4 \delta_{D e f} D_{0} e^{\frac{-E_{D}}{k_{B} T}}}{\xi_{0} e^{-\frac{E_{B}, A}{k_{B} T}}}+1}
$$

From the parameters in equation 11 only $\Gamma, T$ and $\delta_{D e f}$ remain the rest $\gamma, E_{B, A}, D_{0}$ and $E_{D}$ are estimated from MD calculations. Further the Flux $\Gamma$ and the temperature $\mathrm{T}$ are known from experiment leaving $\delta_{D e f}$ as the only free parameter to be used for fitting the model to the experimental data in Fig. 1.

The second model considered here, is the inclusion model. It is based on the idea that energetic particles as they are implanted into the crystal lattice of the target reduce the binding energy through shielding and distorting the lattice. In particular if they are implanted at interstitial positions close to the surface they lower the binding energy of the surface atoms. To calculate the defect sublimation flux for the inclusion model the number of surface atoms with a weakened surface binding energy has to be calculated. In the case of Be erosion by a He plasma He is implanted and then diffuses into the Be target bulk and also towards and out of the surface. Hence in equilibrium there will always be a certain subsurface He concentration depending on the ratio of the incident $\mathrm{He}$ flux to the out-diffusion of He from the Be target as formulated based on Fick's first law in equation 12 :

$$
\begin{aligned}
\left.\rho D(T) \frac{\partial C_{H e}(x, t)}{\partial x}\right|_{x=0} & \equiv(1-r) \Gamma \\
\rho & =\text { Be density } m^{-3} \\
C_{H e}(x, t) & =\text { He concentration } \\
r & =\text { He reflection rate } \\
D(T) & =\text { He in Be } \\
& \text { bulk diffusion }
\end{aligned}
$$


The surface concentration of He at a depth equal to the lattice constant a of Be can be written as a Taylor expansion to first order

$$
C_{H e}(x=a, t)=C_{H e}(0, t)+\frac{\partial C_{H e}(x, t)}{\partial x} a
$$

Due to the inert nature of He each He atom that diffuses to the surface desorbs and thus the surface can be treated as an infinite sink for He. This can be described by a surface boundary condition in the diffusion equation

$$
C_{H e}(0, t) \equiv 0
$$

Using the concentration gradient from equation 12 and the surface He concentration from equation 14 in equation 13 the He subsurface concentration can be written as

$$
C_{H e}(x=a, t)=\frac{(1-r) \Gamma}{\rho D(T)} a
$$

Based on the sub-surface He concentration in equilibrium, the number weakened bonds $\nu$ per subsurface He and the reduced binding energy $E_{B, I}$ of the weakened bonds, the defect sublimation flux can be written as

$$
\begin{aligned}
\Phi_{S u b, A}^{D e f}(\Gamma, T) & =\frac{(1-r) \Gamma}{\rho D(T)} a \nu \delta_{B e} \xi_{0} e^{-\frac{E_{B, I}}{k_{B} T}} \\
\delta_{B e} & =\text { areal density of Be } m^{-3}
\end{aligned}
$$

From the unknown parameters in equation $16, \mathrm{D}(\mathrm{T})$ will be taken from literature data [11], $\mathrm{r}$ for $50 \mathrm{eV}$ He on Be will be calculated using TRIDYN, $\nu$ and $E_{B, I}$ will be estimated from MD-calculations and $\Gamma$ and $\mathrm{T}$ are taken from experiment. Thus no free parameters remain in the inclusion model to fit to the experimental data. 


\section{Molecular dynamics simulations}

The aim of the MD calculations presented here is to obtain reasonable estimates for the input parameters of the above models to decide whether or not they can reproduce the experimental data with a reasonable set of input parameters.

The code used for the molecular dynamics calculations was newly developed for the simulation of impact phenomena during bombardment of solid state surfaces with energetic particles. It was written in $\mathrm{C}++$ and runs either on single CPU environments or on massive multiprocessor environments. The latter version was parallelized using the MPI standard. It can handle amorphous or crystalline solids, $2 \mathrm{D}$ or $3 \mathrm{D}$ periodic boundary conditions and can thus also simulate free surfaces. It supports three different types of temperature control: Simple velocity scaling and coupling to Gaussian [12] or Nose-Hoover [13, 14] type heat baths. The equations of motion are solved using Beemans equations [15] resulting in an $O(\delta 4)$ accuracy in position and $O(\delta 3)$ in velocities. It applies a combination of linked cell and binary space partitioning approach to speed up nearest neighbor search. The use of a binary space partitioning tree allows to quickly cull large portions of the simulation volume which are outside the current potential cutoff resulting in a significant speed increase. For the simulation of energetic particle impact the system can also dynamically adjust the MD time step such that the distance travelled by fastest particle does not exceed a given threshold.

The potentials used in calculations presented here were chosen according to the processes of interest. For the calculation of the ad-atom yield $\gamma$ the important process to simulate was the collision cascade initiated by the impinging energetic particles. Therefore potentials were chosen that modelled the repulsive, high energy part of the Be-Be [16] and He-Be [17] interaction well. For the simulation of processes occurring at thermal energies as the ad-atom surface diffusion and subsurface He inclusion, a Finnis-Sinclair type [18] many body potential was used for the Be-Be [19] interaction and a Born-Mayer type potential for the HeBe [20] interaction. For the calculations presented here a Be crystal was used. 
The parameters of the unit cell used to construct the Be crystal were: $a_{1}=$ $0.22858 \mathrm{~nm}, a_{2}=0.22858 \mathrm{~nm}, a_{3}=0.35843$ and $\measuredangle a_{1}, a_{2}=120^{\circ}, \measuredangle a_{1}, a_{3}=90^{\circ}$, $\measuredangle a_{2}, a_{3}=90^{\circ}$. The unit cell contained two atoms: one at the origin and one at the $\left(\frac{1}{3} a_{1}, \frac{2}{3} a_{2}, \frac{1}{2} a_{3}\right)$ position. A simulation cell containing $11 \times 11 \times 11$ unit cells with an open surface in the $\langle 001\rangle$ direction was used. For the calculation of the ad-atom yield as a function of temperature the impact of $128{ }^{4} \mathrm{He}$ atoms at $50 \mathrm{eV}$ were simulated at 300, 700, 1173 and $1500 \mathrm{~K}$ temperature of the Be simulation cell. The angle of incidence was chosen to be $20^{\circ}$ from the surface normal to avoid channeling of the incident ${ }^{4} \mathrm{He}$. Prior to the impact of each ${ }^{4} \mathrm{He}$ the system temperature was equilibrated during $10^{3}$ steps. While simulating the impact temperature control was turned off. Therefore, the system temperature after the impact, once the system had thermalized, was increased by $\leq 20 \%$. From these calculations the sputter, reflection and ad-atom yield were determined. A simulation cell containing 16x16x5 unit cells with an open surface in the $\langle 001\rangle$ direction and a single ad-atom on the free surface were used for the calculation of the ad-atom diffusion coefficient. Prior to each diffusion measurement the surface temperature was stabilized at the corresponding temperature during $10^{4}$ MD-steps of $2 \mathrm{fs}$ each. Then the brownian motion of the ad-atom was followed for $10^{5}$ MD-steps of $2 \mathrm{fs}$ each at 300, 700, 1173 and 1500K. The surface diffusion coefficient was then determined from the mean square displacement of the ad-atom as in equation 17

$$
D=\frac{\left\langle(r(t)-r(0))^{2}\right\rangle}{4 t}
$$

The average in equation 17 is a time average over all possible time origins. From these calculations also the ad-atom surface binding energy was deduced by averaging the binding energy of the ad-atom over the $10^{5} \mathrm{MD}$-steps that were performed.

To calculate the effect of subsurface He atoms for the inclusion model a Be surface was prepared with He atoms in between the first and second atomic layer. 
The subsurface He atoms were located at unit cell positions that were chosen according to the equilibrium locations of the He atoms implanted during the adatom yield calculations. According to the He in Be bulk diffusion coefficient from [11] an equilibrium subsurface He concentration in order of $1 \%$ is calculated from 15 for an incident flux of $\Gamma=3 \cdot 10^{22} \mathrm{~m}^{-2} \mathrm{~s}^{-1}$. Therefore collective effects (He-He interaction, clustering etc.) are neglected in the models and also in the MD calculations. To determine the reduced binding energy the system was monitored for $10^{3} \mathrm{MD}$-steps of 2 fs at $300 \mathrm{~K}$ Be crystal bulk temperature. Then the binding energies of the atoms in the first atomic layer were averaged over these $10^{3} \mathrm{MD}$-steps. From these averaged binding energies the surface atoms with a reduced binding energy were identified to obtain $\nu$. The reduced binding energy $E_{B, I}$ was then calculated by taking the average reduced binding energy of the $\nu$ surface atoms affected by a single subsurface He inclusion.

\section{Results and Discussion}

In Fig. 2 ad-atom, sputter and reflection yields as a function of temperature are shown for the impact of $50 \mathrm{eV}{ }^{4} \mathrm{He}$ on a Be $\langle 001\rangle$ surface. Also shown for comparison are the sputter and reflection yields calculated using TRIDYN. The sputter and and reflection yields are constant with temperature and compare well to the TRIDYN results. The ad-atom yield is approximately an order of magnitude larger than the sputter yield. The ad-atom yield is not constant with temperature. It approximately doubles at $\approx 800 \mathrm{~K}$ and saturates at $\approx 1200 \mathrm{~K}$. The current working hypothesis to explain this effect is that an activation energy barrier is overcome at $800 \mathrm{~K}$ which results in the observed increase. At higher temperatures the prompt recombination (i.e recombining with bulk material during the duration of the collision cascade) of the created ad-atoms increases, resulting in the saturation of the ad-atom yield.

Fig. 3 shows the calculated surface diffusion coefficient for the Be ad-atoms. Also shown is an arrhenius fit to the data as required in equation 8 . The adatom surface diffusion coefficient is of the order of $10^{-5}$ to $10^{-4} \mathrm{~cm}^{2} \mathrm{~s}^{-1}$ which 
is a reasonable order of magnitude for self-diffusion on metal surfaces.

The calculated surface binding energies of the atoms in the first atomic layer of a Be surface with subsurface He atoms are shown in Fig. 4. The large white circles represent the subsurface He atoms and the small black squares the Be atoms in first atomic layer. The gray scale indicates their surface binding energy in $\mathrm{eV}$. The He atoms are situated at lattice positions identified as their equilibrium positions during the He implantation experiments. Each He atom affects $\nu=3$ surface atoms reducing their binding energy on average to $\approx-2.4$ $\mathrm{eV}$. The influence of each subsurface He atom is sufficiently localized so that there are no cumulative effects on the binding energy of the surface Be atoms. Based on the calculated input parameters for the ad-atom and inclusion model the analytical expressions from section 3 can be compared to the experimental data in Fig. 1. Fig. 5 shows the comparison of the ad-atom model (equation 11 inserted as $\Phi_{\text {Sub }}^{D e f}(T)$ into equation 1) with the He-plasma experimental data in Fig. 1. To calculate the graphs in Fig. 5 the following input parameters were used: $\gamma=0.5, E_{B, A}=2.3 \mathrm{eV}, D_{0}=3 \cdot 10^{-3} \mathrm{~cm}^{2} \mathrm{~s}^{-1}$ and $E_{D}=0.24$ $\mathrm{eV}$ were taken from the MD calculations. $\Gamma=3 \cdot 10^{22} \mathrm{~m}^{-2} \mathrm{~s}^{-1}$ and the surface temperature were taken from the experiment. The remaining free parameter (the areal defect density $\delta_{D e f}$ ) was adjusted to give the best fit to the experimental data. The best fit to the experimental data required $\delta_{D e f} \approx 10^{1} 2 \mathrm{~m}^{-2}$ which corresponds to a required mean distance between two recombination sites of several 1000 lattice spacings. Having such a perfect surface on a non-polished Be target exposed to a He plasma is rather unlikely. On a realistic surface the ad-atoms diffuse to, and recombine with surface defects too fast as to sublimate in sufficient quantities to explain the effect. Therefore ad-atoms are less likely to be the weakly bonded, sublimating surface defect, responsible for the observed increase in the erosion yield.

For the case of He bombardment no free parameters remain to fit the inclusion model (equation 16 inserted as $\Phi_{\text {Sub }}^{D e f}(T)$ into equation 1 ) to the data in Fig. 1. In Fig. 6 the comparison of the inclusion model with the He-plasma experimental data in Fig. 1 is depicted using the following input parameters: $\nu=3$ and 
$E_{B, I}=2.4 \mathrm{eV}$ were taken from the MD calculations. The reflection coefficient $r=0.1$ was taken from a TRIDYN calculation of $50 \mathrm{eV} \mathrm{He}$ on Be at perpendicular angle of incidence. $\Gamma=3 \cdot 10^{22} \mathrm{~m}^{-2} \mathrm{~s}^{-1}$ and the surface temperature were taken from the experiment. The diffusion coefficient of He in Be was taken from [11] and is of the order of $10^{-11} \mathrm{~cm}^{2} \mathrm{~s}^{-1}$ in the temperature range where the thermally enhanced erosion takes place. The inclusion model reproduces the experimental data very well without free parameters. The subsurface He concentration calculated from equation 15 is in the order of $1 \%$. The subsurface He concentration in equilibrium is why this effect cannot be found in low flux ion beam experiments. For lower incident fluxes the subsurface He concentration is basically zero because the out-diffusion of He overcompensates the low incident flux in an ion beam experiment.

Since there are basically no weakly bonded surface atoms, due to the lack of subsurface He atoms, the sublimation rate of weakly bonded surface atoms is now orders of magnitude lower than the normal sublimation rate. Thus the effect cannot be observed at low fluences.

While the He inclusion model very well reproduces the data for He bombardment of Be the question remains, if it can be extended to other projectile/target combinations: for instance the D-plasma data set in Fig. 1. It appears that $\mathrm{D}$ results in a weaker temperature dependence of the erosion yield. This can be understood qualitatively using the inclusion model. D has a much higher out-diffusion rate than He resulting in less subsurface D. Also due to the partly attractive interaction potential between $\mathrm{D}$ and $\mathrm{Be}[17]$ one would expect that subsurface D results in a smaller reduction in binding energy of surface atoms compared to He. These two differences between D and He should result in a weaker temperature increase of the erosion for the $\mathrm{D}$ plasma case.

\section{Conclusions}

Recent experiments show that the erosion rate of metals under high flux low energy particle bombardment exhibits a strong temperature dependence. Using 
the specific example of Be bombardment by He from a He plasma two, analytical models, the ad-atom and He inclusion model, were compared. Generally the intensity of thermally enhanced erosion depends on the interplay of defect production due the high flux particle bombardment and the annealing rate of these defects. The two proposed models are very similar their explanation of the effect. Both rely on the formation and subsequent sublimation of weakly bonded surface defects. In both models these defects are only created in sufficient quantities during bombardment at high particle fluxes due to the fast annealing time scales in metals.

After narrowing down the number of free parameters using MD calculations the ad-atom model has to assume an unrealistically low value for the remaining free parameter (the areal surface defect density) to explain the experimental observations. The recombination rate of the ad-atoms is too high on a realistic surface, making it a less likely explanation. However the contributions from ad-atom sublimation could become significant for cases where the surface diffusion is lower such that the life time of the ad-atoms becomes long enough. This could be the case for heavier substrates like $\mathrm{Au}$ or W.

The He-inclusion model is able reproduce the experimental data without free parameters using the result of the MD calculations. It can in principle be extended to other projectile/target combinations since the implantation of inter-layer, sub-surface atoms usually weakens the binding energy of the surface atoms.

To conclude that the inter-layer, sub surface inclusion of the incident species is in fact responsible for the observed enhancements in erosion, the inclusion model has to be applied to other systems which is currently hampered by the number of available interaction potentials for the MD calculations.

\section{Acknowledgment}

This work was partly funded by the task TW2-TPP-ERDEP of the EFDA technology programme. 


\section{References}

[1] W. Eckstein, C. Garcia-Rosales, J. Roth, W. Ottenberger, Sputtering Data, Vol. 9/82, Max-Planck-Institut für Plasmaphysik, D-85748 Garching, 1993.

[2] W. Eckstein, Computer Simulation of Ion-Solid Interactions, SpringerVerlag, 1991.

[3] N. Bohr, The penetration of atomic particles through matter, Mathematiskfysike Meddelelser 23 (1984) 309.

[4] P. Sigmund, Theory of sputtering. i. sputtering yield of amorphous and polycrystalline targets, Phys. Rev. 184 (1969) 383.

[5] J. Roth, J. Bohdansky, K. L. Wilson, J. Nucl. Mater. 111 \& 112 (1982) 775 .

[6] V. Philipps, K. Flaskamp, E. Vietzke, J. Nucl. Mater. 111 \& 112 (1982) 781.

[7] J. Roth, W. Möller, Nucl. Inst. Meth. B 7/8 (1985) 788.

[8] R. P. Doerner, S. I. Krasheninnikov, K. Schmid, J. Appl. Phys. 95 (2004) 4471.

[9] W. Möller, W. E. und J. P. Biersack, Computer Physics Communications 51 No. 8 (1988) 355.

[10] C. B. Alcock, V. P. Iktin, M. K. Horrigan, Canadian Metallurgical Quaterly 23 (1984) 309.

[11] P. Jung, J. Nucl. Mater. 202 (1993) 210.

[12] M. P. Allen, D. J. Tildesley, Computer simulation of liquids, Clarendon Press, Oxford, 1987.

[13] S. Nose, Mol. Phys. 52 (1984) 255.

[14] W. G. Hoover, Phys. Rev.A 31 (1985) 1695.

[15] D. Beeman, J. Comp. Phys. 20 (1976) 130.

[16] S. Ueda, T. Ohsaka, S. Kuwajima, J. Nucl. Mater. 258 (1998) 71.

[17] S. Ueda, J. Nucl. Mater. 283 (1998) 1100.

[18] M. W. Finnis, J. E. Sinclair, Phil. Mag. A 50 (1984) 45.

[19] M. Igarashi, Phil. Mag. B 63 (1991) 603.

[20] J. M. Cayphas, M. Hou, L. Coheur, J. Nucl. Mater. 246 (1997) 171. 


\section{Figure captions}

Fig. 1

Temperature dependent erosion yield of Be for exposure to a D or He plasma [8]

Fig. 2

Ad-atom, sputter and reflection yield as a function of temperature for the bombardment Be with $50 \mathrm{eV}^{4} \mathrm{He}$ as calculated by MD and TRIDYN

Fig. 3

Surface diffusion coefficient of Be ad-atoms on a Be $\langle 001\rangle$ surface

Fig. 4

Effect of subsurface He atoms on the binding energy of surface atoms

Fig. 5

Comparison of the ad-atom model to the He-plasma experimental data

Fig. 6

Comparison of the He inclusion model to the He-plasma experimental data 
Figure 1:

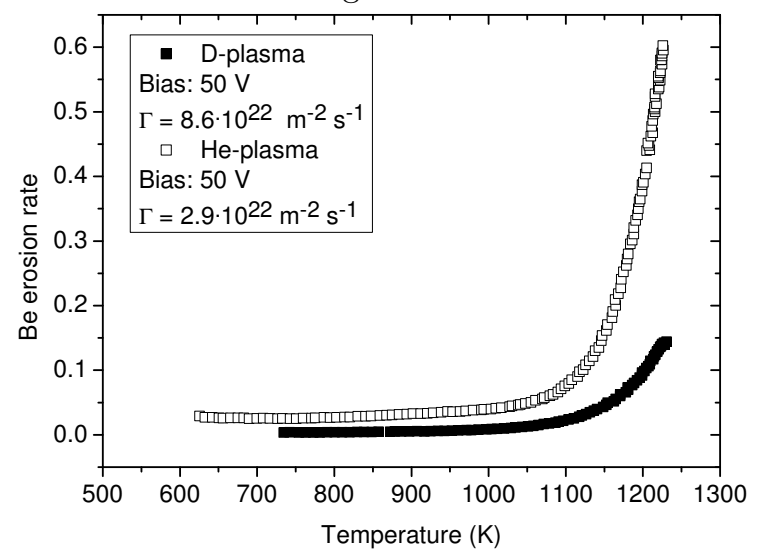

Figure 2:

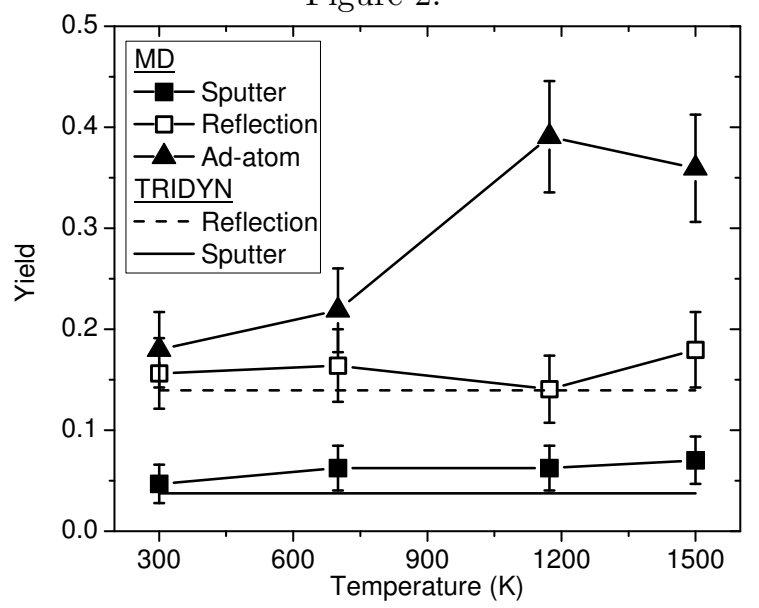


Figure 3:

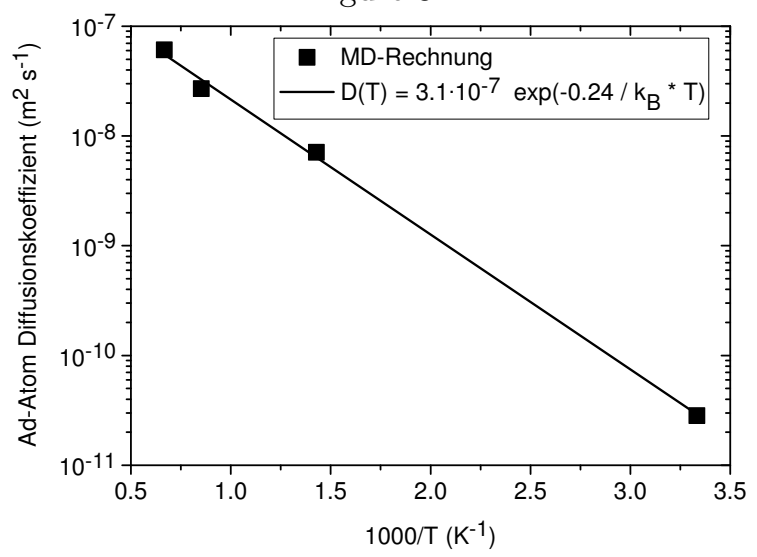

Figure 4:

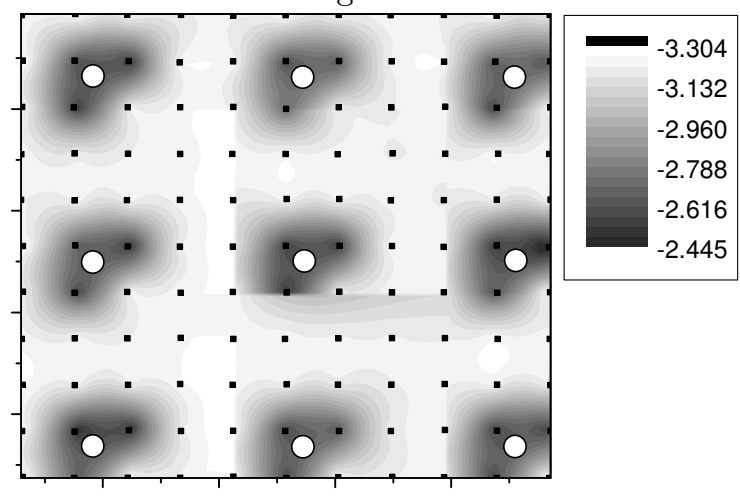

Figure 5:

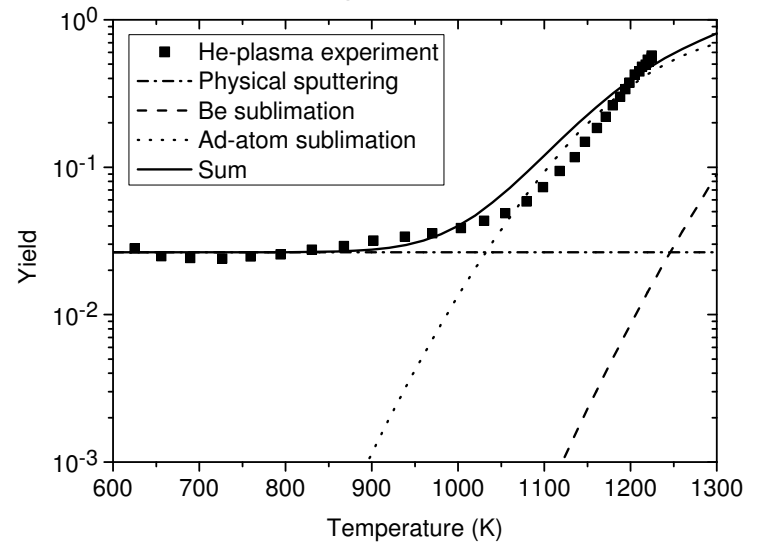


Figure 6:

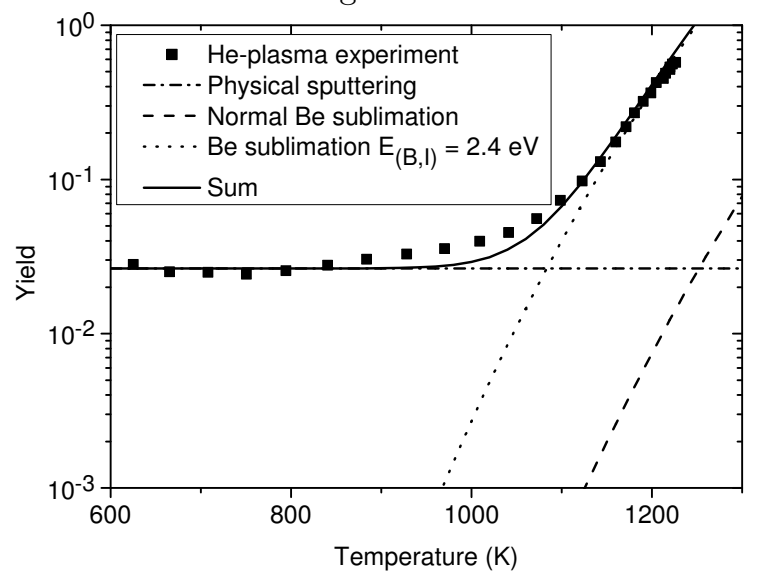

